\title{
Crecimiento primario de Ugni molinae (Myrtaceae) en ARgENTINA: PRODUCCIÓN DE HOJAS, FLORES Y FRUTOS EN DOS POBLACIONES EXPERIMENTALES
}

\author{
Primary growth of Ugni molinae (Myrtaceae) in Argentina: leaf, \\ FLOWER AND FRUIT PRODUCTIONS IN TWO EXPERIMENTAL POPULATIONS
}

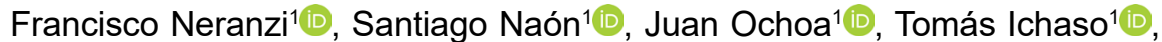 \\ Brisa Guenuleo $^{1}$ D, Sergio Torrego ${ }^{1}$ D, Sonia Stefe ${ }^{1}\left(\mathbb{D}\right.$, Ezequiel Moncunill ${ }^{1}$, \\ Eduardo Martínez ${ }^{1}$ y Javier Puntieri ${ }^{1,2}$ (D)
}

1. Universidad Nacional de Río Negro. Instituto de Investigaciones en Recursos Naturales, Agroecología y Desarrollo Rural (IRNAD), San Carlos de Bariloche, Argentina.

2. Consejo Nacional de Investigaciones Científicas y Técnicas.

*jpuntieri@unrn.edu.ar

Citar este artículo

NERANZI, F., S. NAÓN, J. OCHOA, T. ICHASO, B. GUENULEO, $S$. TORREGO, S. STEFE, E. MONCUNILL, E. MARTÍNEZ \& J. PUNTIERI. 2022. Crecimiento primario de Ugni molinae (Myrtaceae) en Argentina: producción de hojas, flores y frutos en dos poblaciones experimentales. Bol. Soc. Argent. Bot. 57: 25-38.

DOI: https://doi. org/10.31055/1851.2372.v57. n1.35156

Recibido: 13 Oct 2021

Aceptado: 7 Feb 2022

Publicado en línea: 20 Feb 2022

Publicado impreso: 31 Mar 2022

Editora: Ana María Gonzalez iD

ISSN versión impresa 0373-580X

ISSN versión on-line 1851-2372

\section{SUMMARY}

Background and aims:

Ugni molinae (Myrtaceae) is a shrub species that produces berries of excellent aroma taste and nutraceutical properties. It is native to Patagonia, but is barely known in Argentina. The objective of this study was the identification of the pattern of primary growth and of the periods of anthesis and fruit ripening in $U$. molinae plants derived from Argentinean provenances.

M\&M: Through one growth season, the development of 169 one-year-old $U$. molinae plants established in a greenhouse, and 46 four-year-old plants established under open sky was evaluated; all plants had derived from rooted stakes.

Results: All plants developed leaves between mid-spring and late summer. For oneyear-old plants, notable variations were found regarding the time of maximum leaf production and the length of the growth period. The older plants were more regular in their phenologies. Flowering reached its summit between December and January, but the numbers of flowers in anthesis at each single time were low. Fructification levels were high for plants grown under open sky, and nil for greenhouse-grown plants.

Conclusions: One-year-old $U$. molinae plants may exhibit variations regarding the yearly time of maximum growth; four-year-old plants are less variable in this aspect. The development of flowers in a greenhouse is possible, but the presence of pollinators must be ensured for fruit production.

\section{KEY WORDS}

Berries, flowering, growth rate, Myrtaceae, Patagonia, phenology.

\section{RESUMEN}

Introducción y objetivos: Ugni molinae (Myrtaceae) es una especie arbustiva que produce bayas de excelentes aroma, sabor y calidad nutracéutica. Es nativa de Patagonia, pero poco conocida en Argentina. El objetivo principal de este estudio fue determinar el patrón de crecimiento primario y los períodos de antesis y de maduración de frutos de plantas de $U$. molinae de origen argentino.

M\&M: A lo largo de una estación de crecimiento, se documentó el desarrollo de 169 plantas de $U$. molinae de un año en un invernadero, y de 46 plantas de cuatro años establecidas a cielo abierto; todas las plantas derivaron de estacas enraizadas.

Resultados: Todas las plantas produjeron hojas entre mediados de primavera y finales del verano. Para las plantas de un año se registraron variaciones en el tiempo de ocurrencia de la tasa máxima de producción de hojas, y en la duración del crecimiento. Las plantas de mayor edad fueron más regulares en su fenología. La floración alcanzó su nivel máximo entre diciembre y enero, pero los números de flores en antesis en cada momento fueron bajos. Los niveles de fructificación fueron elevados a cielo abierto y nulos en el invernadero.

Conclusiones: Plantas de U. molinae de un año pueden exhibir variaciones en el momento del año de máximo crecimiento primario; las plantas de cuatro años presentan menos variación en este aspecto. Las plantas de U. molinae desarrollan flores en invernadero, pero debe asegurarse la presencia de polinizadores para lograr la fructificación.

\section{Palabras clave}

Fenología, fruta fina, Myrtaceae, Patagonia, tasa de crecimiento. 


\section{INTRODUCCIÓN}

El proceso por el cual una planta produce entrenudos, nudos, hojas y flores a partir de meristemas apicales se conoce como crecimiento primario. La periodicidad o fenología del crecimiento primario de una especie está determinada endógenamente pero su expresión es modulada por factores ambientales; además, puede presentar variaciones intra-específicas e intra-individuales de origen ontogenético (Puntieri et al., 1998; Costes et al., 2006; Sabatier et al., 2003). Conocer la fenología del crecimiento primario y sus variaciones para una especie permite establecer en qué período del año son mayores sus requerimientos ambientales. La fenología del crecimiento primario es bien evidente en especies caducifolias, dado que la expansión de hojas revela el inicio de una fase de alargamiento y su senescencia y caída el inicio de una fase de reposo (Estiarte \& Peñuelas, 2015). En especies perennifolias, el conocimiento de la fenología demanda estudios detallados debido a que los marcadores morfológicos de las interrupciones del crecimiento pueden ser poco obvios (Barthélémy \& Caraglio, 2007). Esta información es fundamental cuando se proyecta incorporar una especie en un agroecosistema, especialmente cuando éste es externo al área de distribución natural de la especie. Si bien la diversificación de los agroecosistemas es muy valorada hoy en día (Lin, 2011; FAO, 2015, 2017), la falta de adecuación de la fenología de una especie a la ventana temporal de condiciones ambientales favorables de la región receptora puede conducir a una merma en rendimiento productivo o a la pérdida del cultivo.

En la actualidad se ha incrementado el interés en el cultivo de especies vegetales con probadas propiedades nutracéuticas y/o medicinales, pero cuyos aprovechamientos actuales son de carácter extractivista (Muñoz et al., 2015; Díaz-Forestier et al., 2019). La zona noroccidental de la Patagonia es una de las regiones de clima templado con mayor diversidad biológica (Rovira et al., 2008). Allí habitan más de 20 especies cuyos frutos tienen largas historias de consumo por parte de pueblos indígenas y pobladores rurales (de Mösbach, 1992; Damascos, 2011). Las áreas de distribución natural de estas especies se han reducido y fragmentado a partir de fines del siglo XIX debido a demandas de uso de la tierra con fines productivos o habitacionales (Folchi, 2015). En la actualidad, los frutos de algunas de ellas son consumidos de forma ocasional por habitantes y visitantes, o recolectados para su comercialización (Muñoz et al., 2015; Ochoa et al., 2019). Pocas especies patagónicas con frutos comestibles han sido recientemente incluidas en sistemas agroproductivos, especialmente en Chile (Beraud \& Tampe Pérez, 2017).

Entre las especies más valoradas en este sentido se encuentra Ugni molinae Turcz. (Myrtaceae), conocida en lengua mapuche como "uñi" y en castellano como "murta" o "murtilla". Este arbusto patagónico de hasta $2 \mathrm{~m}$ de altura tiene valor cultural y comercial como fuente de alimento y por sus propiedades bioactivas asociadas a altos contenidos de compuestos fenólicos, proantocianidinas, flavonoides y carotenoides de sus frutos y hojas (Seguel et al., 1999, 2000, 2009; Águila Chacón \& Nahuelhual Muñoz, 2008; Avello et al., 2009; Delporte et al., 2007; Fredes et al., 2020; López et al., 2018; Suwalsky et al., 2006; Augusto et al., 2014; Junqueira-Goncalves et al., 2015; ArancibiaRadich et al., 2019; Espinoza-Tellez et al., 2021). Los frutos de $U$. molinae fueron y son utilizados por el pueblo mapuche y por otros pobladores rurales de Chile, donde se encuentra gran parte de su área de distribución natural (Landrum, 1988; de Mösbach, 1992; Muñoz et al., 2015). Si bien se dispone de información detallada para su propagación y cultivo en ese país (Doll et al., 2012; Seguel et al., 2009) y en Australia (Forbes-Smith, 2006), hay pocos datos publicados acerca de la fenología de su crecimiento primario (Seguel et al., 1999; Smith-Ramírez et al., 1998).

La distribución de $U$. molinae en Argentina está limitada a la cuenca del Lago Puelo y sus alrededores (mayormente en el Parque Nacional Lago Puelo, PNLP), y su aprovechamiento en zonas rurales es escaso (Ochoa et al., 2019). Con el fin de promover el cultivo de $U$. molinae en Argentina, llevamos adelante un proyecto de investigación del cual ha derivado información referida a sus características morfo-arquitecturales y fenológicas básicas y a su multiplicación. Los primeros resultados de ese proyecto evidenciaron la alta factibilidad de multiplicación de $U$. molinae a partir de semillas o de estacas, con buenas posibilidades de generación en pocos años de individuos bien ramificados y sexualmente maduros 
(Guenuleo et al., 2020). En ese mismo proyecto se dio inicio al cultivo experimental de $U$. molinae tanto fuera como dentro de su área de distribución natural a partir de material vegetativo obtenido en poblaciones nativas de Argentina. El presente aporte tuvo como objetivo principal determinar la fenología del crecimiento primario y los tiempos de antesis y de maduración de frutos de plantas de $U$. molinae de origen argentino. Para ello se establecieron dos estudios independientes. En uno de ellos se evaluaron plantas de un año derivadas de material vegetativo de tres poblaciones naturales establecidas en un invernadero ubicado fuera del área de distribución natural de la especie. El segundo estudio incluyó plantas de cuatro años establecidas a cielo abierto en el PNLP.

\section{Materiales y Métodos}

\section{Poblaciones de estudio}

Estudio 1: En invierno de 2018 se cortaron ramas vigorosas derivadas de la base de plantas de $U$. molinae de tres poblaciones ubicadas dentro de su área natural de distribución en Argentina (Fig. 1). Estas poblaciones se encuentran en el Cordón Derrumbe (CD; 42 09' 56.66" S, 71 ${ }^{\circ} 36^{\prime} 00.26$ " $\mathrm{O}, 354 \mathrm{msnm})$, en la base del cerro Currumahuida (CM; 42 09' 19.26" S, 71³6'40.23” O, 286 $\mathrm{msnm}$ ) y en proximidades del Puerto Silva, sobre la costa Norte del Lago Puelo (PS; 42 ${ }^{\circ} 13^{\prime} 07.61$ " $\mathrm{S}, 71^{\circ} 41^{\prime} 11.03$ " O, $\left.198 \mathrm{msnm}\right)$. A partir de las ramas cortadas se prepararon estacas que fueron enraizadas en cama caliente en el vivero de la Universidad Nacional de Río Negro, ubicado en Bariloche (Río Negro, Argentina; $41^{\circ} 07^{\prime} 37.15^{\prime \prime} \mathrm{S}$, $\left.71^{\circ} 25^{\prime} 30.10^{\prime \prime} \mathrm{O}, 825 \mathrm{msnm}\right)$. Las plantas fueron mantenidas en un invernadero no calefaccionado en macetas de tres litros conteniendo una mezcla de turba, perlita y compost de biosólidos en iguales proporciones. Quincenalmente se aplicó fertilizante orgánico Chase ${ }^{\circledR}(8 \% \mathrm{~N}, 4 \% \mathrm{~K}, 0,8 \% \mathrm{Mn}, 1,4 \% \mathrm{P}$, $0,25 \% \mathrm{~B}, 0,13 \% \mathrm{Cl}, 0,0025 \% \mathrm{Co}, 0,03 \% \mathrm{Cu}, 0,2 \%$ $\mathrm{Fe}, 0,1 \% \mathrm{Zn}, 0,025 \% \mathrm{Mo}$, extracto de algas marinas $10 \%)$. El riego fue aportado por micro-aspersión periódica, en dos turnos diarios de ocho minutos. Se obtuvieron 98 plantas de CD, 41 de PS y 30 de CM; las diferencias entre poblaciones se relacionaron con la cantidad de ramas cortadas de las cuales derivaron estacas con capacidad de enraizamiento.

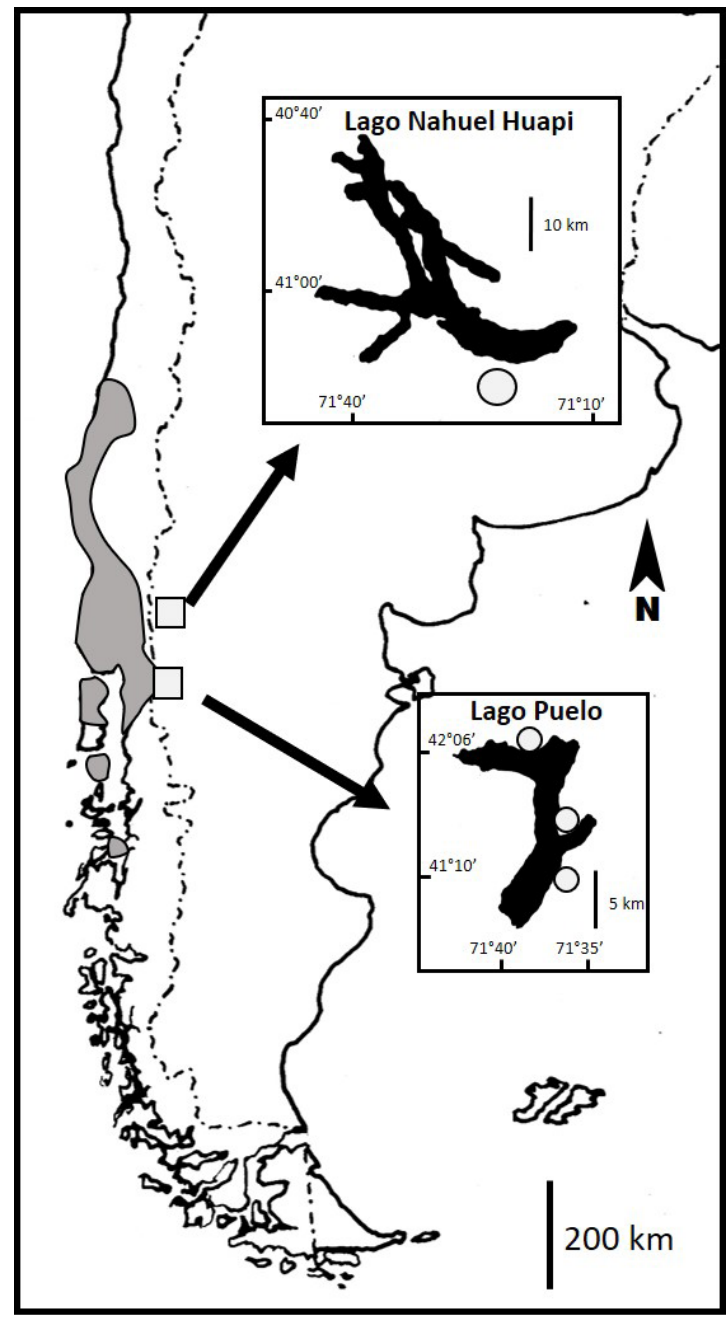

Fig. 1. Distribución natural de Ugni molinae (área en gris, modificado de Landrum, 1988). Se indican las ubicaciones de los lagos Nahuel Huapi y Puelo en el mapa general (cuadrados). En los detalles se ilustran los contornos de esos lagos, y se señalan (círculos) las ubicaciones del invernadero donde se desarrollaron las plantas derivadas de estacas (Bariloche, junto al lago Nahuel Huapi) y de los sitios donde se obtuvieron los esquejes: de norte a sur, Puerto Silva, Currumahuida y Cordón Derrumbe.

Al finalizar la estación de crecimiento 2018-2019 se marcó con una cinta plástica, en cada planta, el brote que había exhibido mayor crecimiento primario en ese período. A partir del 24 de octubre de 2019 se comenzaron a registrar semanalmente los nuevos 
nudos desarrollados a partir de la yema apical de cada uno de los brotes marcados y los números de flores abiertas (en antesis) en cada planta. Una flor fue calificada como "abierta" cuando sus cinco pétalos se encontraban desplegados, dejando visibles al estigma y a los estambres. Los registros de datos se detuvieron en abril de 2020, luego de tres semanas consecutivas sin producción de nuevos nudos en ninguna de las plantas. Mediante marcaciones de hojas distales de los brotes medidos se determinó que no se produjeron hojas nuevas entre abril y septiembre de 2020 .

Estudio 2: Abarcó a 46 plantas de U. molinae de cuatro años establecidas en el PNLP, a menos de $1,5 \mathrm{~km}$ al $\mathrm{E}$ de la población silvestre más próxima. Estas plantas, de entre 20 y $70 \mathrm{~cm}$ de altura y entre 30 y $50 \mathrm{~cm}$ de ancho de copa, habían derivado de estacas obtenidas en 2015 a partir de plantas del PNLP (sin relación a las procedencias del estudio 1$)$, y fueron plantadas en una superficie de $60 \mathrm{~m}^{2}\left(42^{\circ} 05\right.$ ' 21.06" S, 71 $37^{\circ}$ '05.97" O, $202 \mathrm{msnm}$ ), en sustrato compuesto de una mezcla de arena y compost de biosólidos, enriquecida con una dosis de $500 \mathrm{~g}$ de Biobloemen ${ }^{\circledR}$ (abono orgánico concentrado). Estas plantas recibieron riego por goteo. Se aplicó desmalezamiento manual periódicamente. Se verificó el momento de inicio del crecimiento primario de $U$. molinae mediante observaciones semanales a partir de septiembre de 2019 (es decir, en el inicio del quinto período de crecimiento de esas plantas). En la primera semana de octubre de 2019, cuando se observaron los primeros indicios de brotación, dos de los brotes de mayor tamaño desarrollados por cada planta en la estación de crecimiento 2018-2019 fueron marcados con cintas plásticas. Estos brotes fueron: aquel que se encontraba a mayor altura (en adelante "brote alto", BA) y el brote que había alcanzado mayor longitud ("brote largo", BL). Sólo en dos plantas el brote más alto fue el de mayor longitud; en una de ellas ese brote fue considerado BA y en la restante BL. La selección de estos dos brotes por planta se relaciona con el hecho de que los brotes de mayor tamaño en plantas de $U$. molinae que han alcanzado la fase de desarrollo adulta pueden derivar de ejes vigorosos basales o del eje que ha alcanzado mayor altura (Guenuleo et al., 2020). Por esta razón, el vigor de crecimiento de una planta puede estar mejor referenciado por los brotes basales que por los brotes superiores. Ante esta posibilidad, se decidió registrar los crecimientos de ambos brotes. Cada dos o tres semanas se registraron el número de nudos desarrollados a partir de la yema apical de cada uno de los brotes marcados y los números de flores y frutos presentes en cada planta. $\mathrm{Al}$ igual que en el estudio 1 se consignó, en cada fecha de medición, el número de flores abiertas (en antesis) por planta. Las observaciones periódicas se mantuvieron hasta el 31 de marzo de 2020, cuando ya no se observaron cambios en las producciones de nudos de los brotes marcados ni en el inicio de la maduración de nuevos frutos. Se registró el momento de inicio de la maduración de los frutos (cuajado) en base a su coloración, que vira de verde a castaño. Dado que en esta especie los frutos maduros pueden exhibir importantes variaciones de color (entre rosa pálido y rojo oscuro o morado), para determinar el momento de plena maduración se combinaron las percepciones visual, aromática y gustativa.

\section{Análisis de datos}

Para cada uno de los brotes marcados de las plantas de los estudios 1 y 2 , se evaluó la tasa diaria de producción nudos relativa al número de nudos inicial (TPN) mediante la ecuación:

$$
\mathrm{TPN}_{1-0}=\left(\mathrm{n}_{1}-\mathrm{n}_{0}\right) /\left(\mathrm{n}_{0} * \mathrm{t}_{1-0}\right)
$$

(modificada de Květ et al., 1971) donde $\mathrm{n}_{0}$ y $\mathrm{n}_{1}$ son los números de nudos de un brote registrados en los tiempos 0 y 1 respectivamente, $\mathrm{y} \mathrm{t}_{1-0}$ es el número de días transcurrido entre los dos registros. El valor más elevado de TPN de cada planta fue designado TPN máxima $\left(\mathrm{TPN}_{\max }\right.$ ). Para cada planta se determinó la duración del crecimiento estacional de los brotes medidos (período entre el inicio de la brotación y el último registro con TPN $>0$ ).

Los datos de las plantas del estudio 1 fueron agrupados de acuerdo con los patrones generales de las curvas de variación en el tiempo de TPN, siguiendo los ejemplos de trabajos previos (Puntieri et al., 1998, 2018). Las proporciones de brotes de cada procedencia (CD, PS y CM) que se desarrollaron de acuerdo a cada uno de los patrones identificados fueron comparadas mediante una prueba de Chi-cuadrado. La TPN ${ }_{\max }$, la duración del crecimiento y el número de nudos de los brotes medidos fueron comparados entre procedencias y entre grupos mediante pruebas no- 


\section{F. Neranzi et al. - Primary growth of Ugni molinae}

paramétricas de Kruskal-Wallis; las desviaciones de las distribuciones de estas variables respecto de la normalidad (prueba de Kolmogorov-Smirnov) y sus variancias heterogéneas (prueba de Bartlett), aún después de aplicar transformaciones, impidieron el empleo de pruebas estadísticas paramétricas (Sokal \& Rohlf, 1981).

Para el estudio 2, el número de nudos desarrollados por brote se distribuyó normalmente y las variancias fueron similares para BA y BL, por lo cual esa variable se comparó mediante análisis de variancia de una vía y mediante análisis de covariancia (ANCOVA) en el cual se incluyó el tipo de brote como factor fijo y la $\mathrm{TPN}_{\max }$ como covariable. En todas las comparaciones se aplicó un nivel de significación del 5\%.

Para los dos estudios se registraron los números de plantas con producción de hojas nuevas en sus brotes $(\mathrm{TPN}>0)$ y los porcentajes de plantas con flores en antesis en cada una de las fechas de registro.

\section{Resultados}

\section{Estudio 1}

En base a las curvas de variación de la TPN se definieron tres grupos de brotes (GTPN; Fig. 2A-C). GTPN 1: TPN ${ }_{\max }$ al inicio del período de medición sin que los valores posteriores de TPN alcanzaran la mitad de la $\mathrm{TPN}_{\max }$. GTPN 2: $\mathrm{TPN}_{\max }$ registrada al inicio del período de medición, pero con valores posteriores semejantes a la $\mathrm{TPN}_{\max }$. GTPN 3: TPN inicial baja y la $\mathrm{TPN}_{\max }$ registrada luego de transcurridas varias semanas de baja TPN. Para todos los brotes, la TPN descendió al final del mes de diciembre, luego de transcurridas nueve semanas desde el primer registro de datos. Los números de plantas de cada procedencia correspondientes a los GTPN 1, 2 y 3 respectivamente fueron los siguientes: CD: 30,48 y 20 plantas, PS: 14, 15 y 12 plantas y CM: 10,15 y 5 plantas. Las proporciones de brotes correspondientes a cada GTPN fueron similares para las tres procedencias $\left(\mathrm{Chi}^{2}=2,7 ; \mathrm{p}>0,1\right)$.
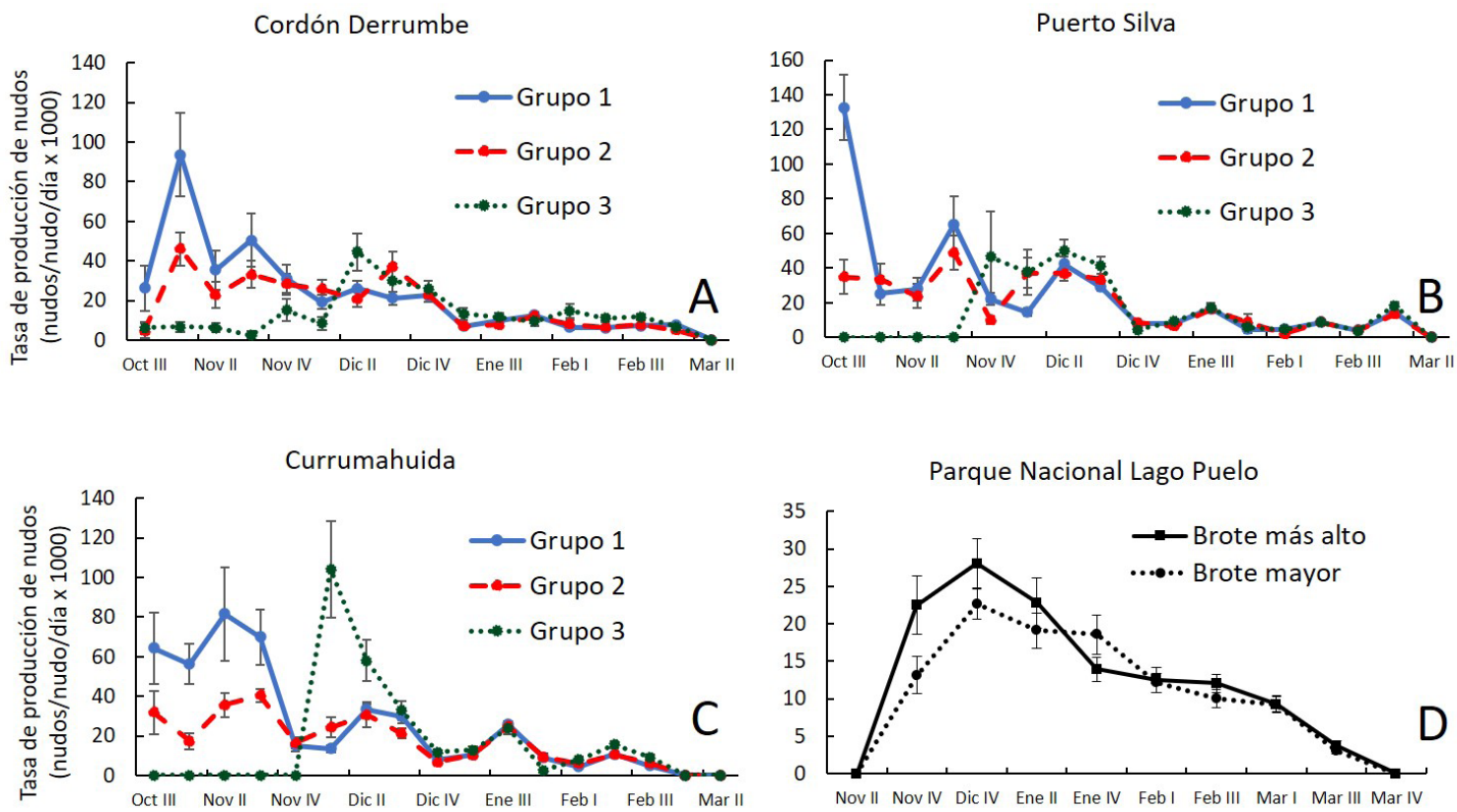

Fig. 2. Variación a lo largo de la estación de crecimiento de la tasa relativa de producción de nudos (TPN; media \pm 1 error estándar) para plantas desarrolladas en invernadero derivadas de estacas a partir de ramas obtenidas en los sitios Cordón Derrumbe (A), Puerto Silva (B) y Currumahuida (C), y de plantas desarrolladas a cielo abierto en el Parque Nacional Lago Puelo (D). En el eje de abscisas se indican mes y número de semana del mes (I, II, III, IV). En A, B y C se diferencian las plantas con tres patrones diferentes de variación de la tasa de producción de nudos (Grupos 1, 2 y 3). En D se diferencian los brotes correspondientes a los ejes ubicados a mayor altura en las plantas y los brotes de los ejes de mayor tamaño. 
La TPN $_{\max }$ varió de forma significativa entre GTPN (prueba de Kruskal-Wallis, $\mathrm{H}=51,4$; $\mathrm{p}<0,001)$ pero no entre procedencias $(\mathrm{H}=1,4$; $\mathrm{p}>0,1)$. La mediana de la TPN ${ }_{\max }$ fue mayor para los brotes del GTPN 1 (0,14 nudos/nudo/día) que para los brotes de los GTPN 2 y 3 (0,08 nudos/nudo/ día en ambos $G T P N$ ). La mayoría de los brotes de las tres procedencias y de los tres GTPN se encontraban en crecimiento en los períodos entre la segunda mitad de diciembre y la primera de enero, $\mathrm{y}$ entre la segunda mitad de febrero y la primera de marzo (Fig. 3A-C). Entre esos dos períodos (segunda mitad de enero a primera de febrero) la mayoría de los brotes no produjeron hojas nuevas.

La duración del crecimiento fue menor para los brotes del GTPN 3 que para los de GTPN 1 y $2(\mathrm{H}=$ 47,$1 ; p<0,001 ;$ Fig. 4). No se registraron diferencias significativas en la duración del crecimiento entre procedencias $(\mathrm{H}=1,0 ; \mathrm{p}>0,1)$. El número de nudos desarrollados al final de la estación de crecimiento difirió entre $\operatorname{GTPN}(\mathrm{H}=28,8 ; \mathrm{p}<0,001)$ : la mediana fue mayor para GTPN 1, 19 nudos, que para GTPN 2, 18 nudos, y GTPN 3, 14 nudos. También se registraron diferencias en número de nudos entre procedencias $(H=11,9 ; p<0,01)$ : la mediana fue mayor para la procedencia CM, 19 nudos, que para PS y CD, 17 nudos en ambos casos. Para las tres procedencias se verificó una relación positiva entre el número de nudos desarrollados por un brote y la duración de su alargamiento, aunque con importante dispersión para valores de duración elevados (Fig. 5).

El porcentaje de plantas que desarrollaron flores fue del $26,5 \%$ para la población CD y del $36,6 \%$ para la población PS. Ninguna de las plantas de la población $\mathrm{CM}$ floreció durante el estudio. La proporción de plantas que florecieron fue menor para GTPN 3 que para GTPN 1 y $2\left(\mathrm{Chi}^{2}=8,43\right.$, $\mathrm{p}<0,05)$. El mayor porcentaje de flores en antesis ocurrió en la primera mitad de diciembre (Fig. 6A). En ninguna de las plantas de este estudio se completó la maduración de frutos.
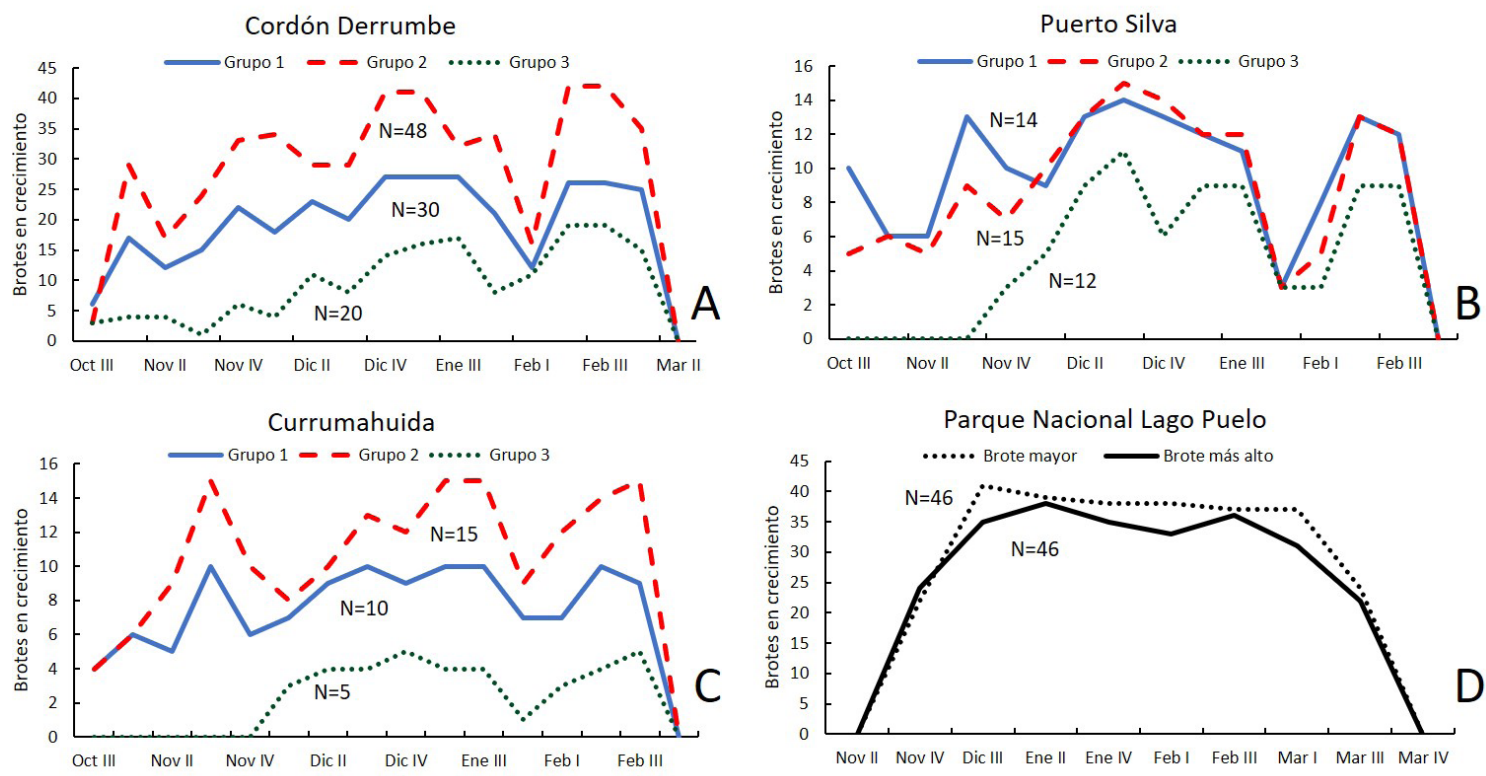

Fig. 3. Variaciones en el número de brotes en crecimiento a lo largo del período de estudio para plantas desarrolladas en invernadero derivadas de estacas a partir de ramas obtenidas en los sitios Cordón Derrumbe (A), Puerto Silva (B) y Currumahuida (C), y de plantas desarrolladas a cielo abierto en el Parque Nacional Lago Puelo (D). En el eje de abscisas se indican mes y número de semana del mes (I, II, III, IV). En A, B y C se diferencian las plantas con tres patrones diferentes de variación de la tasa de producción de nudos (Grupos 1, 2 y 3). En D se diferencian los brotes correspondientes a los ejes ubicados a mayor altura en las plantas y los brotes de los ejes de mayor tamaño. Se indican los números de brotes correspondientes a cada línea $(\mathrm{N})$. 


\section{F. Neranzi et al. - Primary growth of Ugni molinae}
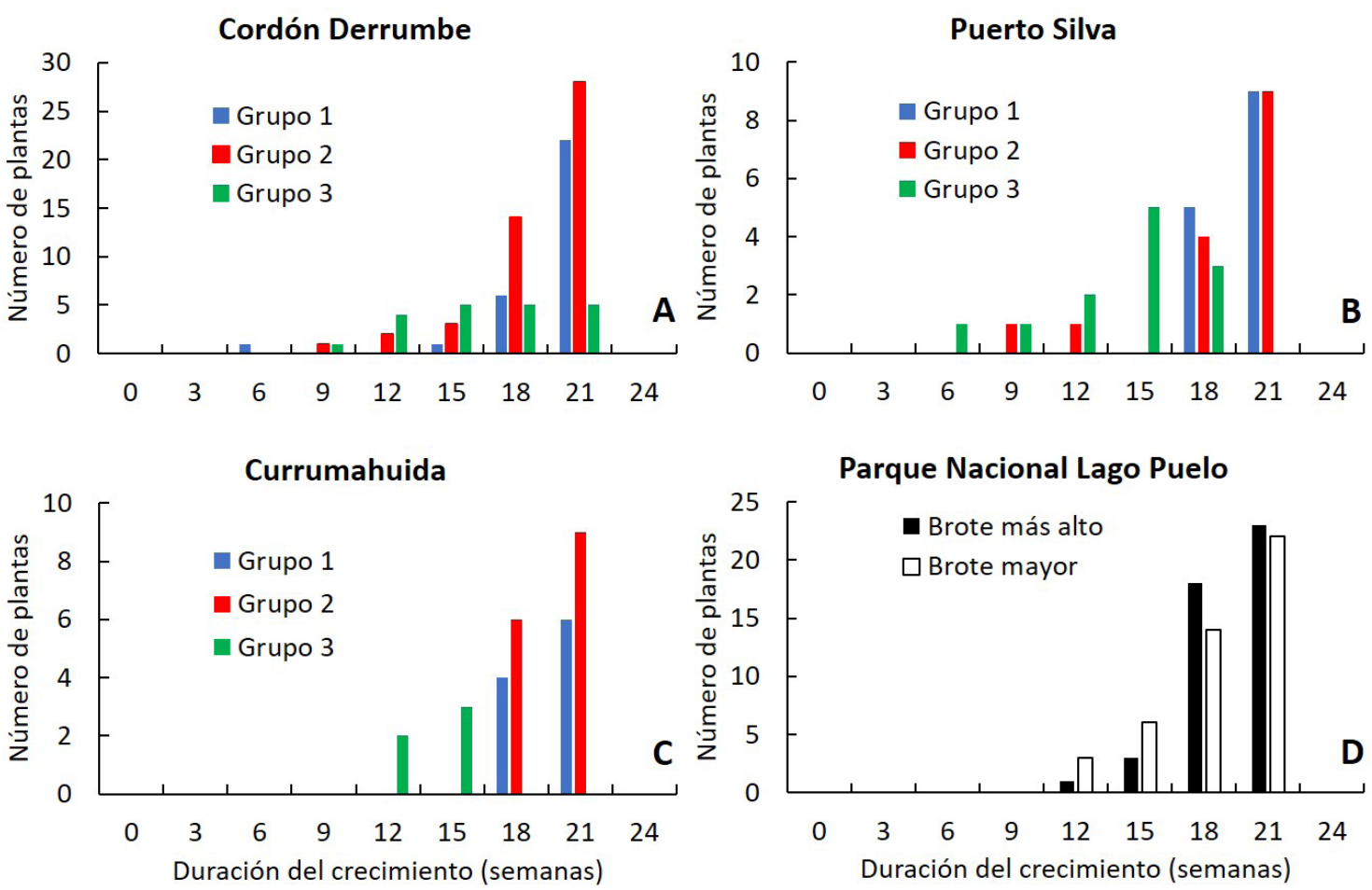

Fig. 4. Distribuciones de frecuencias de las duraciones del crecimiento de los brotes de plantas de Ugni molinae desarrolladas en invernadero derivadas de estacas de los sitios Cordón Derrumbe (A), Puerto Silva (B) y Currumahuida (C), y de plantas desarrolladas a cielo abierto en el Parque Nacional Lago Puelo (D). En A, B y C se diferencian las plantas con tres patrones diferentes de variación de la tasa de producción de nudos (Grupos 1, 2 y 3). En D se diferencian los brotes correspondientes a los ejes ubicados a mayor altura en las plantas y los brotes de los ejes de mayor tamaño.

\section{Estudio 2}

La TPN $_{\text {max }}$ se registró dentro de los primeros dos meses y medio de alargamiento (entre noviembre $\mathrm{y}$ enero) tanto en BA como en BL (Fig. 2D). La comparación de $\mathrm{TPN}_{\max }$ entre $\mathrm{BA}$ y $\mathrm{BL}$ con la prueba de Kruskal-Wallis mostró diferencias significativas (BA $>\mathrm{BL} ; \mathrm{H}=7,9, \mathrm{p}<0,05$ ). Para la mayoría de los brotes no se detectaron períodos intermedios de interrupción del alargamiento (Fig. 3D). El crecimiento primario de los brotes marcados se produjo a lo largo de un período máximo de 20 semanas; algunos brotes completaron su alargamiento en alrededor de 12 semanas (Fig. 4D).

El promedio de número de nudos desarrollados por los brotes marcados fue semejante en BL y BA (medias de 17,4 y 15,4 nudos, respectivamente; $\mathrm{F}$ $=3,5 ; \mathrm{p}>0,05)$. Mediante ANCOVA se determinó que el número de nudos desarrollados por brote no dependió de la $\operatorname{TPN}_{\max }(\mathrm{F}=1,9 ; \mathrm{p}>0,1)$, pero sí del tipo de brote considerado $(\mathrm{F}=4,9 ; \mathrm{p}<0,05)$; para igual $\mathrm{TPN}_{\max }$, los $\mathrm{BA}$ desarrollaron mayor número de nudos al final de la estación de crecimiento que los BL. La duración del crecimiento fue similar para los $\mathrm{BL}$ (mediana $=18$ semanas) que para los BA (20 semanas; $\mathrm{H}=0,45 ; \mathrm{p}>0,05)$. El número de nudos de un brote se relacionó positivamente con la duración de su crecimiento (Fig. 5).

Para esta población se registraron flores en antesis entre los meses de diciembre y enero (Fig. 6A). En cada momento de observación los números de flores en antesis fueron bajos en relación con los números totales de estructuras reproductivas en desarrollo, lo cual determinó que los porcentajes de plantas con flores en antesis fueran también bajos $(<30 \%)$ pese a que todas las plantas desarrollaron flores y frutos (Fig. 6A). Los frutos comenzaron a tomar el color indicativo del inicio de la maduración en el mes de enero, y alcanzaron el color, el aroma 
Bol. Soc. Argent. Bot. 57 (1) 2022

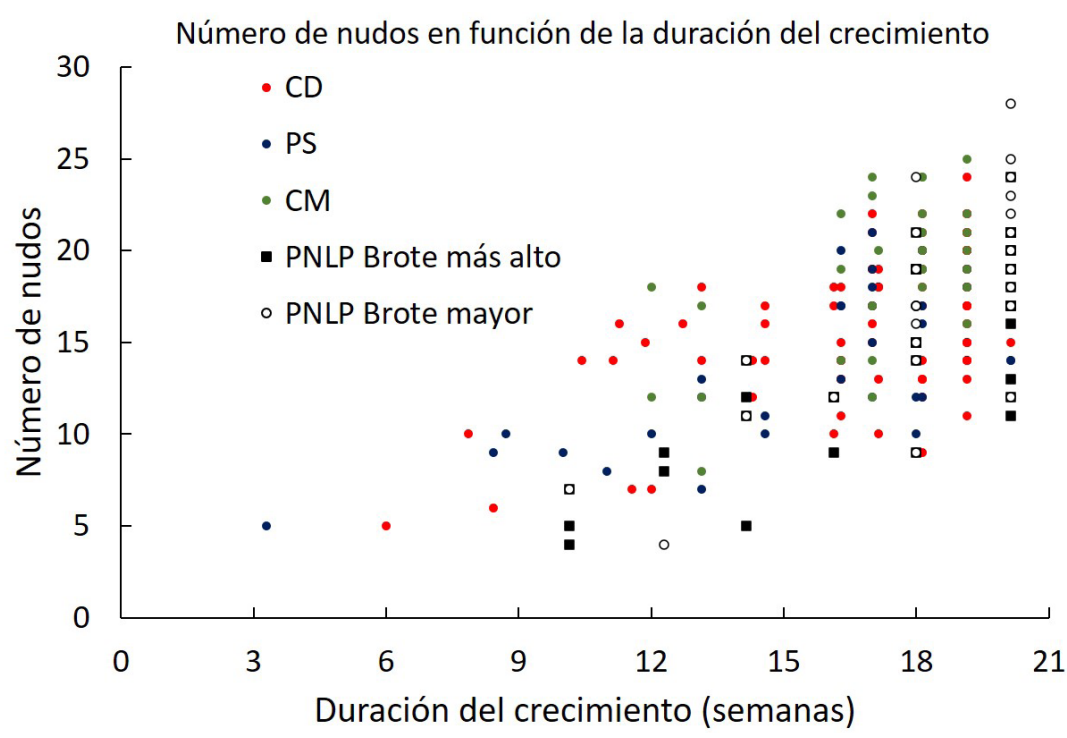

Fig. 5. Relación entre el número de nudos desarrollados y la duración del período de crecimiento por brote para las plantas derivadas de estacas de los sitios Cordón Derrumbe (CD), Puerto Silva (PS) y Currumahuida (CM), y para las plantas desarrolladas en el Parque Nacional Lago Puelo (PNLP, diferenciando brote mayor y brote más alto).

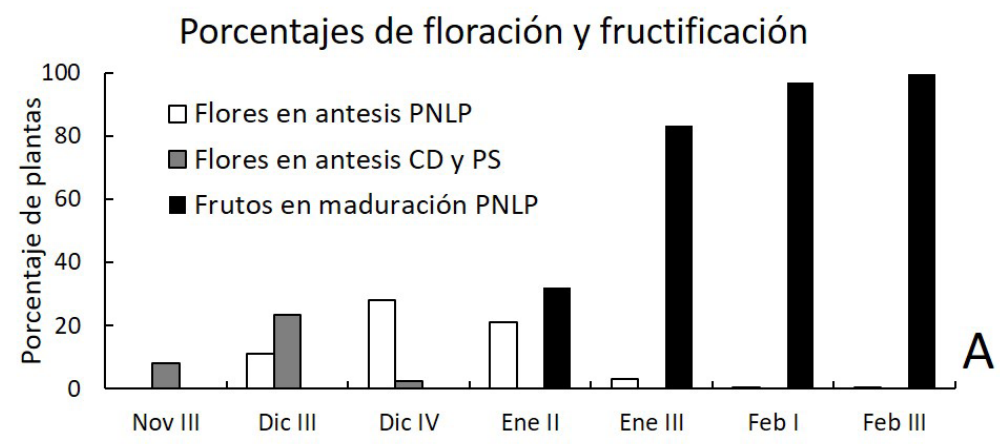

Números de flores y frutos

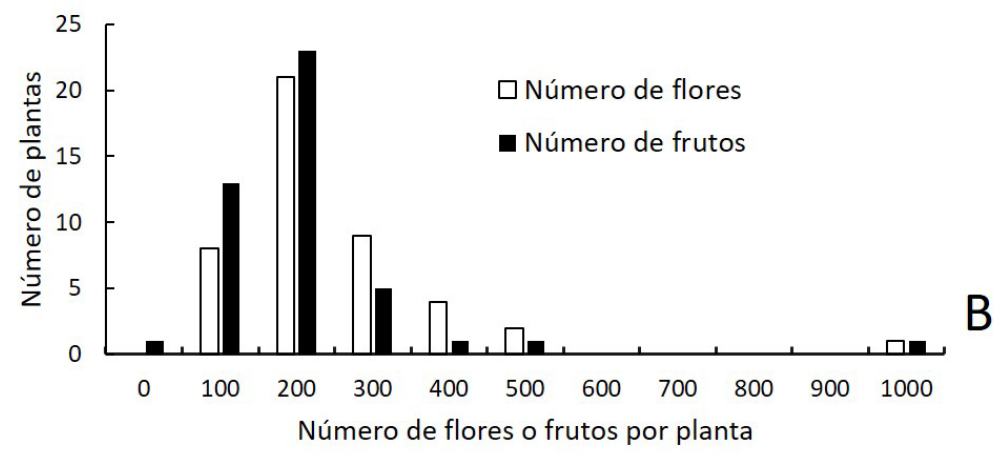

Fig. 6. A: Variaciones a lo largo de la estación de crecimiento de los porcentajes de plantas con flores en antesis en el Parque Nacional Lago Puelo (PNLP) y en invernadero (procedencias Cordón Derrumbe, CD, y Puerto Silva, PS), y de los porcentajes de plantas con frutos en desarrollo en el PNLP. B: Distribuciones de frecuencias de los números totales de flores y de frutos por planta en el PNLP. 


\section{F. Neranzi et al. - Primary growth of Ugni molinae}

y el gusto que indican maduración plena en el mes de febrero (entre tres y cuatro meses luego del inicio de la brotación). Se cosecharon frutos maduros entre uno y dos meses después del inicio de la coloración de los frutos. La mayoría de las plantas desarrollaron entre 100 y 300 frutos, en concordancia con las sumas de flores registradas (Fig. 6B).

\section{Discusión}

Tasa máxima, duración y plasticidad del crecimiento en brotes de U. molinae

En este trabajo se evaluaron la fenología y el patrón de crecimiento primario para plantas de $U$. molinae de dos edades (uno y cuatro años) derivadas de estacas y desarrolladas en condiciones contrastantes. Para las plantas de las dos edades y condiciones, el período de crecimiento primario de los brotes abarcó entre fines de octubre y marzo, y se extendió, como máximo, por alrededor de 20 semanas. Tanto la fenología como la duración del crecimiento registradas aquí para $U$. molinae son comparables, en términos generales, con las obtenidas para brotes vigorosos de otras especies leñosas de Patagonia (Puntieri et al., 1998, 2018; Salgado et al., 2020). Se destaca como diferencia notable entre las plantas de $U$. molinae de las dos edades la variabilidad en el patrón de crecimiento primario. Entre las plantas de un año dicha variabilidad fue alta debido a las diferencias (dentro de cada procedencia de plantas) en cuanto al momento en que la tasa de producción de nudos alcanzó su valor máximo. La mayoría de los brotes de las plantas de un año presentaron la tasa máxima de producción de nudos al inicio de la estación de crecimiento, seguida por un descenso en el crecimiento (GTPN 1 y 2). La mayoría de los brotes en los cuales la tasa máxima de producción de nudos fue tardía (GTPN 3) iniciaron su brotación alrededor de un mes más tarde que los demás brotes. Por otro lado, el crecimiento de los brotes de las plantas de cuatro años desarrolladas en el PNLP presentó siempre una tasa máxima temprana, que fue menor que la registrada en las plantas de un año. En las plantas de cuatro años, los brotes ubicados a mayor altura y los derivados del eje más vigoroso de la planta (en general no localizado en el ápice de la copa), se asemejaron en sus patrones de crecimiento a los brotes de los GTPN 1 y 2, respectivamente, de las plantas de un año, aunque presentaron tasas de crecimiento menores que éstos. Los brotes vigorosos no apicales compensaron la menor tasa de crecimiento máxima con un menor descenso de la tasa de crecimiento luego de alcanzado ese máximo.

Independientemente de la edad de las plantas y del momento en que ocurre la tasa máxima de crecimiento, los brotes de $U$. molinae pueden continuar produciendo nuevas hojas durante la segunda mitad del verano, aunque con tasas de producción relativamente bajas. Dicha producción de hojas prolonga la duración del crecimiento e incide significativamente en el tamaño de los brotes al final de su alargamiento.

Los menores niveles de variación en la producción de nudos y las menores tasas máximas de producción de nudos en las plantas de cuatro años desarrolladas en el PNLP en relación con las plantas de un año podrían relacionarse con la fase ontogenética más regular y avanzada de las primeras. Es frecuente que las plantas en instancias más avanzadas de su ontogenia expresen menor variabilidad en la estructura de sus brotes (Barthélémy \& Caraglio 2007). Para la mayoría de las especies patagónicas estudiadas hasta el momento se observaron, aún para ejemplares juveniles, altos niveles de homogeneidad intraespecífica en cuanto a los tiempos de brotación estacional y de tasa máxima de alargamiento (Puntieri et al., 1998, 2018; Stecconi et al., 2000, 2017; Salgado et al., 2020), en tanto que para pocas especies (Schinus patagonica (Phil.) I.M. Johnst. y Maytenus boaria Mol.) se registraron variaciones notables en el inicio de la brotación y en el período de ocurrencia de la tasa máxima de producción de hojas (Salgado et al., 2020).

Las variaciones fenológicas a niveles intrapoblacional y/o intra-individual revelan diferencias funcionales entre ejes que inciden en la arquitectura de las plantas (Costes et al., 2006) y que pueden resultar beneficiosas ante cambios imprevisibles en las condiciones ambientales. Por ejemplo, aquellos ejes en los cuales se demora la expansión de las hojas podrían evitar la exposición a heladas tardías, en tanto que aquellos ejes cuyos crecimientos primarios finalizan tempranamente tendrían un tiempo de rustificación más prolongado antes de las heladas de otoño. La plasticidad en términos de producción de órganos les permite a las plantas enfrentar condiciones ambientales sub-óptimas 
(Henn et al., 2018; Pfennigwerth et al., 2017), e incrementa las probabilidades de aclimatación de una especie a sitios fuera de su área de distribución natural.

Si bien todas las plantas utilizadas en este estudio derivaron de estacas producidas a partir de ramas obtenidas de varias poblaciones naturales, los resultados indican que el desarrollo a lo largo de cuatro años desde el enraizamiento sería suficiente para que las plantas de $U$. molinae alcanzaran la instancia de desarrollo no jerárquico, en el cual el sistema de ramas se compone de ejes orientados verticalmente y con brotes de tamaños similares (Guenuleo et al., 2020). En esta fase de desarrollo la floración se presenta en toda la copa, tanto en ramas largas como en ramas cortas, y se inicia la formación de brotes vigorosos derivados de yemas cercanas al suelo o subterráneas (derivadas de rizomas; Guenuleo et al., 2020). En esta instancia del desarrollo de $U$. molinae, sería pertinente, dada la densidad de la ramificación basal y con miras al cultivo de esta especie para la producción de frutos, el raleo de ramas vigorosas basales para favorecer el acceso de los polinizadores a las flores y a la cosecha de los frutos. Las podas basales de ejemplares con características deseables pueden ser aprovechadas para multiplicarlos por medio de estacas.

\section{Variaciones intra-estacionales en las tasas de crecimiento}

Uno de los factores que pueden incidir en las variaciones en la tasa de crecimiento de un brote es el número de primordios de la yema precedente a ese brote, es decir, el nivel de preformación. Altos niveles de preformación determinan el alargamiento simultáneo de mayor número de órganos, lo cual se traduce en altas tasas de crecimiento al producirse la brotación de primavera (Puntieri et al., 1998). Por otro lado, el hecho de que la tasa máxima de producción de hojas ocurra en etapas más avanzadas de la estación de crecimiento indica la prevalencia de neoformación de órganos sobre la preformación (Guédon et al., 2006). Para algunas especies es sabido que las variaciones en el número de hojas preformadas en las yemas de invierno se relacionan con las fases ontogenéticas en las que se encuentran una planta y los ejes que la componen (Puntieri et al., 2000, 2007; Sabatier et al., 2003; Souza et al., 2000). Es decir que las variaciones en la dinámica de producción de hojas de los brotes de $U$. molinae podrían evidenciar diferencias ontogenéticas entre los brotes medidos.

La producción de hojas en las plantas de $U$. molinae de un año descendió notablemente entre fines de enero y principios de febrero, y ascendió a mediados de febrero (Fig. 3). Los descensos estivales en la producción de hojas han sido mencionados para otras especies leñosas de la Patagonia andina de Argentina (Puntieri et al., 1998; Stecconi et al., 2000; Sosa \& Puntieri, 2016; Salgado et al., 2020), y si bien se los podría vincular con factores ambientales (temperatura y/o disponibilidad de agua), hasta el momento no se han encontrado evidencias contundentes en ese sentido. En $U$. molinae, el descenso estival en la tasa de crecimiento puede reconocerse a posteriori por la presencia de un par de hojas que, aunque se reconocen como nomofilos por su contorno y consistencia, tienen menor tamaño que las hojas previas y las hojas subsiguientes (J. Puntieri, obs. pers.). Este patrón de crecimiento primario se asemeja al crecimiento calificado como "bicíclico" que se observa en especies de los géneros Cedrus, Juglans, Pinus y Quercus, entre otros, aunque en esos casos el biciclismo implica una detención más prolongada entre picos de alargamiento y mayor nivel de diferenciación foliar entre los dos pulsos, por la formación de catafilos que cubren la yema terminal entre los pulsos (Sabatier et al., 2003; Cline \& Harrington, 2007).

\section{Desarrollo de flores y frutos}

El aporte principal de este trabajo en cuanto a la floración y la fructificación de $U$. molinae procede de las plantas desarrolladas en el PNLP, la mayoría de las cuales desarrollaron estructuras reproductivas en sus estaciones de crecimiento cuarta y quinta. El período de floración registrado aquí comprendió, principalmente, los meses de diciembre y enero, en concordancia con la información disponible para Chile (Smith-Ramírez et al., 1998; Nissen et al., 2010). Los primordios florales en U. molinae derivan de los primeros nudos desarrollados durante el alargamiento de un brote y continúan formándose a partir de nudos subsiguientes del mismo brote, lo cual determina cierto grado de amplitud fenológica en la floración de cada planta e incluso de un mismo brote (Guenuleo et al., 2020), tal como ocurre en Luma apiculata (DC.) Burret, otra especie de 


\section{F. Neranzi et al. - Primary growth of Ugni molinae}

Myrtaceae de Patagonia (Puntieri et al., 2018). Por otro lado, los bajos porcentajes de plantas de $U$. molinae con flores en antesis en cada momento de observación (Fig. 6A) se explican por el corto período en que cada flor permanece abierta. Los números de flores y frutos en cada una de estas plantas presentaron alto nivel de correspondencia, lo cual sugeriría que la presencia de insectos polinizadores en esa población fue adecuada, habida cuenta del bajo nivel de autopolinización que se presenta en esta especie (Neira et al., 2003).

Respecto de las plantas de un año (estudio 1) fueron muy notables las diferencias entre procedencias en cuanto al desarrollo de flores: presentes en las plantas de las procedencias Puerto Silva y Cordón Derrumbe y ausentes en las de Currumahuida. Es muy probable que esas diferencias se deban a la condición de las plantas cuyas ramas fueron cortadas para formar las estacas. Esto explicaría el hecho de que los brotes de Currumahuida desarrollaran mayor número de nudos que los de las otras dos procedencias. Los cambios en la morfología de los brotes desarrollados por un eje en fases sucesivas de la secuencia ontogenética de su especie, suelen incluir el incremento en el desarrollo de estructuras reproductivas y la merma en el crecimiento vegetativo (e.g. Caesar \& Macdonald, 1983). Por ello, podría asumirse que los ejes de Currumahuida de los cuales derivaron las estacas habrían estado en una fase ontogenética menos avanzada que los de las restantes procedencias. La dependencia de $U$. molinae respecto de los insectos polinizadores (Neira et al., 2003) explicaría la falta de cuajado de frutos en el invernadero.

En este estudio registramos un lapso de alrededor de dos meses entre el pico de floración y la maduración de los frutos, en concordancia con los resultados reportados por Smith-Ramírez et al. (1998) para Chile. Considerando las demás especies de mirtáceas patagónicas, ese lapso es intermedio entre los de especies con frutificación más próxima a la floración (como L. apiculata) y los de especies con floración y fructificación más distanciadas entre sí (como Myrceugenia spp.; Smith-Ramírez et al., 1998). Cabe destacar que los frutos inmaduros de $U$. molinae adquieren forma y coloración semejantes a las de los frutos maduros (rojo o castaño oscuro) cerca de un mes antes de alcanzar el aroma y el sabor que los distingue.

\section{Conclusiones}

Si bien el crecimiento primario de $U$. molinae se produce principalmente en el período primaveraverano, pueden encontrarse variaciones notables respecto del momento de tasa de crecimiento primario máxima en plantas de un año derivadas de estacas. Luego de cuatro/cinco años desde el enraizamiento de estacas, los brotes desarrollados por las plantas presentan menores niveles de variación en su crecimiento primario $\mathrm{y}$ buen desarrollo de flores $\mathrm{y}$, potencialmente, de frutos (que depende de la disponibilidad de polinizadores). La floración alcanza su nivel máximo entre fines de diciembre y principios de enero. La permanencia de cada flor en antesis es relativamente corta. La maduración de los frutos, que insume cerca de dos meses desde el pico de floración, debe verificarse por la percepción de tamaño, color, aroma y gusto.

\section{Contribución de los Autores}

FN, SN y JGP realizaron el mantenimiento y los registros de datos de las plantas establecidas en Bariloche. Todos los co-autores evaluaron el crecimiento de las plantas del Parque Nacional Lago Puelo y contribuyeron en la redacción y corrección del texto.

\section{Agradecimientos}

Este estudio fue financiado por la Universidad Nacional de Río Negro, Sede Andina (PI UNRN 40-B-663). Los autores agradecen la colaboración del personal de la Intendencia del Parque Nacional Lago Puelo en el riego de las plantas de U. molinae allí establecidas.

\section{Bibliografía}

ÁGUILA CHACÓN, C. \& L. NAHUELHUAL MUÑOZ. 2008. Cultivo de murtilla (Ugni molinae Turcz.) como alternativa de diversificación productiva para la agricultura familiar campesina de la Cordillera de la Costa. Agro Sur 36: 158-167. https://doi.org/10.4206/agrosur.2008.v36n3-05 
ARANCIBIA-RADICH， J., R. GONZÁLEZBLÁZQUEZ, M. ALCALÁ, M. MARTÍN-RAMOS, M. VIANA, S. ARRIBAS, C. DELPORTE, M. FERNÁNDEZ-ALFONSO, B. SOMOZA \& M. GIL-ORTEGA. 2019. Beneficial effects of murtilla extract and madecassic acid on insulin sensitivity and endothelial function in a model of diet-induced obesity. Sci. Rep. 9: 599.

https://doi.org/10.1038/s41598-018-36555-1

AUGUSTO, T.R., E.S. SCHEUERMANN SALINAS, S.M. ALENCAR, M.A. D'ARCE, A. COSTA DE CAMARGO \& T.A. VIEIRA. 2014. Phenolic compounds and antioxidant activity of hidroalcoholic extracts of wild and cultivated murtilla (Ugni molinae Turkz.). Food Sci. Technol. 34: 667-673. https://doi.org/10.1590/1678-457X.6393

AVELlO, M., R. VALDIVIA, R. SANZANA, MA. MONCADA, S. MENNICKENT, V. AESCHLIMANN, M. BINTTNER \& J. BECERRA. 2009. Extractos antioxidantes y antimicrobianos de Aristotelia chilensis y Ugni molinae y sus aplicaciones como preservantes en productos cosméticos. Bol. Latinoamericano y del Caribe de Plantas Medicinales y Aromáticas 8: 469-478.

BARTHÉlÉMY, D. \& Y. CARAGLIO. 2007. Plant architecture: a dynamic, multilevel and comprehensive approach to plant form, structure and ontogeny. Ann. Bot. 99: 375-407.

https://doi.org/10.1093/aob/mcl260

BERAUD, M.R. \& J. TAMPE PÉREZ. 2017. Efectos del ácido giberélico, N6-bencilaminopurina y fluridona sobre la germinación in vitro de Aristotelia chilensis. Bosque 38: 593-597. https://doi.org/10.4067/S0717-92002017000300017

CAESAR, J.C. \& D. MACDONALD. 1983. Shoot development in Betula papyrifera. II. Comparison of vegetative and reproductive short-shoot growth. Canad. J. Bot. 61: 3066-3071. https://doi.org/10.1139/b83-343

CLINE, M.G. \& C.A. HARRINGTON. 2007. Apical dominance and apical control y multiple flushing of temperatewoodyspecies.Canad.J.For.Res. 37:74-83. https://doi.org/10.1139/x06-218

COSTES, E., P.-E. LAURIE \& J.L. REGNARD. 2006. Analyzing fruit tree architecture: implications for tree management and fruit production. Horticultural Reviews 32, JULES JANICK (ed.).

DAMASCOS, M.A. 2011. Arbustos silvestres con frutos carnosos de Patagonia. Calidad - Propiedades Usos. Fondo Editorial Rionegrino, Viedma.
DELPORTE, C., N. BACKHOUSE, V. INOSTROZA, M.C. AGUIRRE, N. PEREDO, X. SILVA, R. NEGRETE \& H.F. MIRANDA. 2007. Analgesic activity of Ugni molinae (murtilla) in mice models of acute pain. J. Ethnopharmacol. 30: 162-165. https://doi.org/10.1016/j.jep.2007.02.018

DE MÖSBACH, E.W. 1992. Botánica Indígena de Chile. Editorial Andrés Bello, Santiago de Chile.

DÍAZ-FORESTIER, J, P. LEÓN-LOBOS, A. MARTICORENA, J.L. CELIS-DIEZ \& P. GIOVANNINI. 2019. Native Useful Plants of Chile: A Review and Use Patterns. Econ. Bot. 20: 1-15. https://doi.org/10.1007/s12231-019-09447-2

DOLL, U., I. RODRÍGUEZ, C. SOTO \& I.C. RAZMILIC. 2012. Propagación de estacas y concentración de taninos y flavonoides en hojas de dos procedencias de Ugni molinae de la región del Maule (Chile). Bosque 32: 203-209.

https://doi.org/10.4067/S0717-92002012000200010

ESPINOZA-TELLEZ, T., J.M. BASTÍAS-MONTES, R. QUEVEDO-LEÓN, E. VALENCIA-AGUILAR, O. DÍAZ-CARRASCO, M.A. SOLANOCORNEJO \& F. MESA-MESINA. 2021. The murta (Ugni molinae) and its beneficial health properties: A review. Sci. Agropecuaria 12: 121-131. https://doi.org/10.17268/sci.agropecu.2021.14

ESTIARTE, M. \& J. PEÑUELAS. 2015. Alteration of the phenology of leaf senescence and fall in winter deciduous species by climate change: effects on nutrient proficiency. Global Change Biol. 21: 10051017. https://doi: $10.1111 / \mathrm{gcb} .12804$

FAO. 2015. Climate change and food security: risks and responses. Food and Agriculture Organization of the United Nations, Roma.

FAO. 2017. The future of food and agriculture - Trends and challenges. Food and Agriculture Organization of the United Nations, Roma.

FORBES-SMITH, M. 2006. Tazziberry ${ }^{\mathrm{TM}}$ (Myrtus ugni) - Production protocols. Rural Industries Research and Development Corporation, Sydney.

FREDES, C., A. PARADA, J. SALINAS \& P. ROBERT. 2020. Phytochemicals and traditional use of two southernmost Chilean berry fruits: murta (Ugni molinae Turcz) and calafate (Berberis buxifolia Lam.). Foods 9: 54.

https://doi:10.3390/foods9010054

FOLCHI, M. 2015. Historia de las áreas protegidas en Chile. En: Simonetti-Grez, G., J.A. Simonetti \& G. Espinoza (Eds.). Conservando el Patrimonio Natural de Chile: el Aporte de las Áreas Protegidas, 


\section{F. Neranzi et al. - Primary growth of Ugni molinae}

pp. 33-60. Programa de las Naciones Unidas para el Desarrollo, Santiago de Chile.

GUÉDON, Y., J. PUNTIERI; S. SABATIER \& D. BARTHÉLÉMY. 2006. Relative extents of preformation and neoformation in tree shoots: analysis by a deconvolution method. Ann. Bot. 98: 835-844. https://doi.org/10.1093/aob/mcl164

GUENUlEO, B., S. TORREGO, S. STEFE, F. NERANZI, E.L.N. MONCUNILL, S. NAÓN, M.L. CARDOZO, E. MARTÍNEZ, J.J. OCHOA \& J. PUNTIERI. 2020. Características morfoarquitecturales y fenológicas de Ugni molinae (Myrtaceae): una especie arbustiva andinopatagónica con frutos comestibles. Bol. Soc. Argent. Bot. 55: 215-230.

https://doi.org/10.31055/1851.2372.v55.n2.26034

HENN J.J., V. BUZZARD, B.J. ENQUIST, A.H. HALBRITTER, K. KLANDERUD, B.S. MAITNER, S.T. MICHALETZ, C. PÖTSCH, L. SELTZER, R.J. TELFORD, Y. YANG, L. ZHANG \& V. VANDVIK. 2018. Intraspecific trait variation and phenotypic plasticity mediate Alpine plant species response to climate change. Front. Plant Sci. 9: 1548. https://doi:10.3389/fpls.2018.01548

JUNQUEIRA-GONCALVES, M. P., L. YANEZ, C. MORALES, M. NAVARRO, R.A. CONTRERAS \& G.E. ZUNIGA. 2015. Isolation and characterization of phenolic compounds and anthocyanins from murta (Ugni molinae Turcz.) fruits. Assessment of antioxidant and antibacterial activity. Molecules 20: 5698-5713.

https://doi.org/10.3390/molecules20045698

KVĚT, J., J.P. ONDOK, J. NEČAS \& P.G. JARVIS. 1971. Methods of growth analysis. In: SESTÁK, Z., J. CATSKÝ \& P.G. JARVIS (Eds.). Plant Photosynthetic Production, Manual of Methods, pp. 343-391. Juek NV Pub., The Hague.

LANDRUM, L.R. 1988. The myrtle family (Myrtaceae) in Chile. Calif. Acad. Sci. 45: 277-317.

LIN, B. 2011. Resilience in agriculture through crop diversification: adaptive management for environmental change. BioScience 61: 183-193. https://doi.org/10.1525/bio.2011.61.3.4

LÓPEZ J., A. VEGA-GÁLVEZ, A. RODRÍGUEZ, E. URIBE \& C. BILBAO-SAINZ. 2018. Murta (Ugni molinae Turcz.): a review on chemical composition, functional components and biological activities of leaves and fruits. Chilean J. Agric. Anim. Sci. 34: $1-14$.

https://doi.org/10.4067/S0719-38902018005000205
MUÑOZ M., D. AEDO \& J. SAN MARTÍN. 2015. Antecedentes sobre la recolección y comercialización de productos forestales no madereros (PFNM), en localidades rurales de la región del Maule, Chile central. Bosque 36: 121-125.

https://doi.org/10.4067/S0717-92002015000100013

NEIRA M., E.R. SYLVESTER, M. RIVEROS, R. CARRILLO \& C. CÁRDENAS. 2003. Biología reproductiva y entomofauna asociada a las flores de murta (Ugni molinae Turcz.) y evaluación del comportamiento de los Himenópteros polinizadores. Revista Chilena Entomol. 29: 71-79.

NISSEN M.J., G.M. DEL RÍO, I.B. SEGUEL \& B.L. TORRALBO. 2010. Efecto de diferentes manejos hídricos sobre el crecimiento vegetativo y reproductivo de murtilla (Ugni molinae Turcz). Agro Sur 38: $42-54$.

https://doi.org/10.4206/agrosur.2010.v38n1-05

OCHOA, J.J., E.L.N. MONCUNILL, J.G. PUNTIERI, B.S. GUENULEO, S.E. STEFE, M.L. CARDOZO, F. NERANZI BARRIGA, E.E. MARTÍNEZ, S. TORREGO \& S. NAÓN. 2019. Saberes locales y frutos comestibles de plantas nativas en la Comarca Andina del Paralelo $42^{\circ}$ (Patagonia, Argentina). Ethnoscientia 4: 1-9. https://doi.org/10.22276/ethnoscientia.v4i1.247

PFENNIGWERTH, A.A., J.K. BAILEY \& J.A. SCHWEITZER. 2017. Trait variation along elevation gradients in a dominant woody shrub is populationspecific and driven by plasticity. AoB PLANTS 9: plx027. https://doi:10.1093/aobpla/plx027

PUNTIERI, J., D. BARTHÉLÉMY, P. MARTINEZ, E. RAFFAELE \& C. BRION. 1998. Annual-shoot growth and branching patterns in Nothofagus dombeyi (Fagaceae). Canad. J. Bot. 76: 673-685. https://doi.org/10.1139/b98-041

PUNTIERI, J., M. S. SOUZA, D. BARTHÉLÉMY, C. BRION, M. NUÑEZ \& C. MAZZINI. 2000. Preformation, neoformation and shoot structure in Nothofagus dombeyi (Nothofagaceae). Canad. J. Bot. 78: 1044-1054.

https://doi.org/10.1139/b00-069

PUNTIERI, J., J.E. GROSFELD, M. STECCONI, C. BRION \& D. BARTHÉLÉMY. 2007. Bud and growth-unit structure in seedlings and saplings of Nothofagus alpina (Nothofagaceae). Amer. J. Bot. 94: 1382-1390. https://doi.org/10.3732/ajb.94.8.1382

PUNTIERI, J., C. TORRES, A. MAGNIN, M. STECCONI \& J.E. GROSFELD. 2018. Structural differentiation 
among annual shoots as related to growth dynamics in Luma apiculata trees (Myrtaceae). Flora 249: 86-96. https://doi.org/10.1016/j.flora.2018.10.005

ROVIRA, J., J. UGALDE \& M. STUTZIN. 2008. Biodiversidad de Chile. Patrimonio y Desafios. Ocho Libros Editores Ltda., Santiago de Chile.

SABATIER, S., D. BARTHÉLÉMY \& I. DUCOUSSO. 2003. Periods of organogenesis in mono- and bicyclic annual shoots of Juglans regia L. (Juglandaceae). Ann. Bot. 92: 231-238. https://doi.org/10.1093/aob/mcg127

SALGADO, M., C.D. TORRES, A. MAGNIN, M. GEREA, J.E. GROSFELD, J. PUNTIERI \& M. STECCONI. 2020. The dynamics of primary growth in woody species from rain and transitional forests of Argentinean north Patagonia. Bosque 41: 183-192. https://doi.org/10.4067/S0717-92002020000200183

SEGUEL, I., M. RIVEROS, C. LEHNEBACH \& A. TORRES. 1999. Antecedentes fenológicos y reproductivos de Ugni molinae Turcz. (Myrtaceae). Phyton 65: 13-21.

SEGUEL, I., E. PEÑAlosA, N. GAETE, A. MONTENEGRO \& A. TORRES. 2000. Colecta y caracterización molecular de germoplasma de murta (Ugni molinae Turcz.) en Chile. Revista Agro Sur 28: 32-41.

https://doi.org/10.4206/agrosur.2000.v28n2-05

SEGUEL, I., L. TORRALBO, E. PEÑALOZA, E. SCHEUERMANN, A. MONTENEGRO, A. FRANCE, K. SHUNG, J. SAN MARTÍN \& N. ESPINOSA. 2009. Murtilla parte 1: La gran promesa de la fruticultura chilena. Tierra Adentro 87: 49-51.

SMITH-RAMÍREZ C., J.J. ARMESTO \& J. FIGUEROA. 1998. Flowering, fruiting and seed germination in Chilean rain forest myrtaceae: ecological and phylogenetic constraints. Plant Ecol. 136: 119-131. https://doi.org/10.1023/A:1009730810655

SOKAL, R.R. \& F.J. ROHLF. 1981. Biometry. The Principles and Practice of Statistics in Biological Research. 2nd. ed. W.H. Freeman \& Company, New York.

SOSA, C. \& J. PUNTIERI. 2016. Crecimiento anual de cuatro especies arbóreas con yemas desnudas nativas de los bosques templados húmedos de Patagonia. Bol. Soc. Argent. Bot. 51: 643-655.

https://doi.org/10.31055/1851.2372.v51.n4.16341

SOUZA, M. S., J. PUNTIERI, D. BARTHÉLÉMY \& C. BRION. 2000. Bud leaf primordia content and its relation to shoot size and structure in Nothofagus pumilio (Poepp. et Endl.) Krasser (Nothofagaceae). Ann. Bot. 85: 547-555.

https://doi.org/10.1006/anbo.1999.1097

STECCONI, M., J. PUNTIERI \& D. BARTHÉLÉMY. 2000. Annual shoot-growth in Nothofagus antarctica (G. Forster) Oersted (Fagaceae) from northern Patagonia. Trees 14: 289-296. https://doi.org/10.1007/PL00009770

STECCONI, M., L. QUEVEDO, A. MAGNIN, C. TORRES, K. LEDIUK, M. SVRIZ, J. GROSFELD \& J. PUNTIERI. 2017. Desarrollo de especies leñosas nativas y exóticas en cipresales patagónicos. Bol. Soc. Argent. Bot. 52: 507-522. https://doi.org/10.31055/1851.2372.v52.n3.18030

SUWALSKY, M., P. ORELLANA, M. AVELLO, F. VILLENA \& S.P. SOTOMAYOR. 2006. Human erithrocytes are affected in vitro by extracts of Ugni molinae leaves. Food Chem. Toxicol. 44: 1393-1398. https://doi.org/10.1016/j.fct.2006.03.003

WEINBERGER, P. 1978. Estudio sobre adaptación climática y las asociaciones de las Mirtáceas Arauco-Patagónicas. Anales de Parques Nacionales 14: 133-160. 